\title{
Neighborhood Social Cohesion and Inequalities in COVID-19 Diagnosis Rates by Area-Level Black/African American Racial Composition
}

\author{
Yusuf Ransome (iD - Bisola O. Ojikutu • Morgan \\ Buchanan • Demerise Johnston • Ichiro Kawachi
}

Accepted: 11 February 2021 / Published online: 23 March 2021

(C) The New York Academy of Medicine 2021

\begin{abstract}
Geographic inequalities in COVID-19 diagnosis are now well documented. However, we do not

Y. Ransome $(\bowtie) \cdot$ M. Buchanan

Department of Social and Behavioral Sciences, Yale School of Public Health, 60 College Street, LEPH 4th Floor, New Haven, CT 06510, USA

e-mail: yusuf.ransome@yale.edu

B. O. Ojikutu

Division of Infectious Diseases, Brigham and Women's Hospital, Boston, MA, USA
\end{abstract}

B. O. Ojikutu

Infectious Disease Division, Massachusetts General Hospital, Boston, MA, USA

B. O. Ojikutu

Division of Global Health Equity, Harvard Medical School, Boston, MA, USA

B. O. Ojikutu

Department of Medicine, Brigham \& Women's Hospital, Boston, MA, USA

D. Johnston

Department of Environmental Health Sciences, Yale School of Public Health, New Haven, CT, USA

I. Kawachi

Department of Social and Behavioral Sciences, Harvard T.H.

Chan School of Public Health, Boston, MA, USA sufficiently know whether inequalities are related to social characteristics of communities, such as collective engagement. We tested whether neighborhood social cohesion is associated with inequalities in COVID-19 diagnosis rate and the extent the association varies across neighborhood racial composition. We calculated COVID-19 diagnosis rates in Philadelphia, PA, per 10,000 general population across 46 ZIP codes, as of April 2020. Social cohesion measures were from the Southeastern Pennsylvania Household Health Survey, 2018. We estimated Poisson regressions to quantify associations between social cohesion and COVID-19 diagnosis rate, testing a multiplicative interaction with Black racial composition in the neighborhood, which we operationalize via a binary indicator of ZIP codes above vs. below the city-wide average (41\%) Black population. Two social cohesion indicators were significantly associated with COVID-19 diagnosis. Associations varied across Black neighborhood racial composition ( $p<0.05$ for the interaction test). In ZIP codes with $\geq 41 \%$ of Black people, higher collective engagement was associated with an $18 \%$ higher COVID-19 diagnosis rate (IRR $=1.18,95 \% \mathrm{CI}=1.11,1.26)$. In contrast, areas with $<41 \%$ of Black people, higher engagement was associated with a $26 \%$ lower diagnosis rate $(\mathrm{IRR}=0.74,95 \% \mathrm{CI}=0.67$, 0.82). Neighborhood social cohesion is associated with both higher and lower COVID-19 diagnosis rates, and the extent of associations varies across Black neighborhood racial composition. We recommend some strategies for reducing inequalities based on the segmentation model within the social cohesion and public health intervention framework. 
Keywords Racial disparities · Inequality · COVID-19 • Black/African American · Ecological · Spatial .

Structural determinants $\cdot$ Social cohesion $\cdot$ Social capital

Racial and geographic inequalities in confirmed COVID19 cases in the USA are now well established [1, 2]. For instance, in Pennsylvania (PA), Black people comprise $11 \%$ of the population but accounted for $28 \%$ of COVID19 diagnosed cases as of May 28, 2020. Considering geography, the extent of Black-White differences in diagnosis rates varies widely across counties $[1,2]$.

Inequalities in COVID-19 infections are heavily influenced by factors indicative of structural racism [3] (e.g., area-level socioeconomic deprivation and racial residential segregation) [4] that disproportionally exacerbated exposure risk in predominantly Black areas [5, 6]. Structural racism factors also influence the pathways and probabilities of people engaging and maintaining adherence to primary prevention strategies (e.g., wearing masks consistently, washing hands, practicing social distancing), which may vary by the geographic profile of the neighborhood [7].

A major priority for health inequalities research currently is to identify which social characteristics of communities influence inequalities in COVID-19 diagnosis [8]. Social capital and cohesion are social characteristics of communities $[9,10]$. Social capital can be defined broadly as collective resources available through social connections or networks that individuals or groups can access, as well as features of organizations (e.g., trust in others and group norms) that make it possible to achieve coordinated action [11]. Social cohesion describes the cognitive aspects of social capital such as perceptions of trust, feelings of belongingness, and reciprocity which are related to structural aspects such as working to improve one's community and other forms of neighborhood collaboration [12].

Socially and psychologically experienced characteristics of the neighborhood environment (e.g., close contact with people, degree of trust for one's neighbor) may influence COVID-19 transmission dynamics, both positively and negatively. For example, social participationone indicator of the construct - might be positively associated with COVID infections if people in those areas continue high levels of in-person engagements and ignore social distancing practices. Feelings of belongingness - a second indicator - might be associated with lower COVID infections in areas with lower scores. It is plausible that lower cognitive scores at the neighborhood level are indicative of social isolation rates which are correlated with lower in-person contact, which may lower exposure rates. Another pathway through which higher feelings of belongingness may be associated with lower COVID infections is through strong neighborhood attachment. In areas where people have strong attachments to their community, there might be higher motivation and reason to practice COVID prevention protocols to protect one's neighbors.

Empirically, social capital has been linked with previous SARS-related infectious diseases. In one study, social capital and cohesion indicators - civic participation and trust in government-were associated with higher likelihoods of wearing a face mask and washing hands more frequently during the influenza pandemic [13]. In another study, neighborhood social cohesion moderated the impact of H1N1-related knowledge and likelihood of a parent getting their children vaccinated for H1N1 [14]. There still only a handful of studies that assessed social cohesion and COVID-19-related outcomes [15-19]. In one study, among adults in the country of Colombia, the authors found that that low social capital was associated with higher perceived stress related to COVID-19 [20]. Wu conducted a multilevel study among individuals and provinces in China and found that higher collective efficacy among individuals was associated with lower odds of knowing someone infected with COVID-19 [15]. In one US study, the authors found that counties with higher bonding social capital scores had lower excess COVID-19 deaths over three time periods during the pandemic [21]. There is still a dearth of research on this topic in the US context. Previous US-based studies have mainly been countylevel ecological studies. To our knowledge, no studies have examined these associations at the local or smaller geographic resolutions like ZIP codes within a city. To address these gaps, we investigated whether neighborhood social cohesion is associated with COVID-19 diagnosis rates in the population. Results may inform components of COVID-19 prevention strategies at the local level.

Inequalities in COVID-19 diagnosis are patterned by the racial composition within an area [22]. It is therefore plausible that the size and direction of associations between social cohesion and COVID-19 diagnosis rates will vary across distributions of Black neighborhood composition. Briefly, in the US, the Black racial composition of neighborhoods is a reliable proxy indicator of the impact of structural racism's effects on 
geographic communities through legacies of slavery, which has been described as a fundamental cause of racial disparities [23]. Residential segregation (and other) structural racism factors in predominantly Black geographic communities thwarted wealth generation and produced high-income inequality [24] and social exclusion, which erodes social cohesion $[9,25]$. Thus, how social capital is accumulated and how social cohesion is practiced will vary depending on the level of neighborhood racial composition [26]. We therefore investigate whether any association between social cohesion and COVID-19 diagnosis rate varies at distributions above and below the city-wide average Black racial composition.

\section{Methods}

\section{Study Population}

We used population-based COVID-19 counts of positive and negative cases reported by the Philadelphia Department of Public Health. Data are publicly available on Open Data Philly [27], which contained deidentified aggregated data at the ZIP code level only $(N=46)$, as of April 24, 2020, 11:21 AM.

Ethical Statement

Informed consent was not required. These data were not considered human subjects research and exempt from institutional IRB.

Measures

COVID-19 Diagnosis Rate Is the Primary Outcome of the Study

COVID-19 diagnosis rates using confirmed positive cases were calculated per 10,000 general population.

\section{Social Cohesion Is the Primary Exposure Variable in the Study}

The Southeastern Pennsylvania Household Health Survey (SPHHS) 2018-2019 provided data on five social cohesion indicators. Briefly, the SPHHS is a randomdigit dialing comprehensive telephone and cellphone survey that assesses population responses to health, social, and behavioral items among approximately 7500 adults age 18 years and older in Bucks, Chester, Delaware, and Philadelphia counties [28]. Data are weighted to the census population and can be analyzed at the ZIP code level. Data were restricted to Philadelphia County where the average (over the five indicators) number of respondents who provided to calculate social cohesion was $n=3447$. The five indicators were the following: (1) feelings of belongingness, derived from the question "I feel that I belong and am a part of my neighborhood?"; (2) trust in neighbors, derived from the question "most people in my neighborhood can be trusted"; (3) neighbors willing to help, derived from the question "please rate how likely people in your neighborhood are willing to help their neighbors with routine activities such as picking up their trash cans, or helping to shovel snow"; (4) civic and social participation is a count variable corresponding to the question "how many local groups or organizations in your neighborhood do you currently participate in such as social, political, religion, school-related, or athletic organization?"; and (5) collective engagement, from the question "have people in your neighborhood ever worked together to improve the neighborhood?". Following the Empirical Bayes Estimation technique in prior work [29], each indicator was estimated using survey-weighted linear, logistic, or negative binomial regression as appropriate for the distribution and adjusted for the following covariates (race/ethnicity, age, sex, education, marital status, home ownership). The adjusted predicted estimates were aggregated to the ZIP code level. This adjusted approach minimizes any potential confounding in estimates of social cohesion. However, this covariateadjusted score, even with individual race in the model, does not affect the statistical relationship in this ecological-level analysis where area-level racial composition was included.

Black Neighborhood Racial Composition Is the Secondary Exposure Variable This measure was operationalized as the proportion of the population that are Black or African American alone in 2019.

\section{Covariates}

ZIP code level data for select demographics for year 2019 were retrieved from the SimplyAnalytics database online. Selected demographics and other covariates for multivariable analyses were informed by prior research 
on neighborhood social cohesion and other social determinants of COVID-19 and other infectious diseases like HIV [22, 30, 31]. These included a principal component index of neighborhood socioeconomic position comprised of median household income, proportion of residents with a high school diploma, and proportion of residents with no car (Cronbach's $\alpha=0.86$ ). Next was the proportion of people between ages 18 to 24 and 25 to 34; the proportion of renters that moved in 2010 or later; and a crime index, which is a weighted composite of murder, forcible rape, robbery, aggravated assault, burglary, larceny, and motor vehicle theft. A COVID-19 testing site variable was included as a covariate. However, data on number of tests performed at specific sites or within ZIP codes are not available. Addresses for the COVID-19 testing sites in Philadelphia, PA, as of May 2020 were provided by the Philadelphia Department of Public Health. Addresses were geocoded in ESRI Arc GIS Pro software. Kernel density estimates were calculated via spatial statistics, and density scores were assigned to each ZIP code using the zonal statistics to table tool. Residuals of COVID-19 rates were included as a covariate in multivariable regression to adjust for potential spatial autocorrelation of COVID infection patterns. The variable was created via spatial clustering techniques using Queen Contiguity Neighborhood Matrix with $2^{\text {nd }}$ Order structure in GeoDA software.

\section{Statistical Analysis}

Predictor variables were standardized to have a mean of zero and standard deviation of one (i.e., z-scored). Spearman correlation among all the variables was conducted to inform model specification. Exploratory variance inflation tests (VIF) indicated that removing trust and neighbors willing to help would result in the best and parsimonious statistical model. Subsequently, those two variables were removed from the bivariate models and multivariable interaction model.

Descriptive statistics were conducted to describe the median and interquartile range of COVID-19 diagnosis across ZIP codes stratified by areas above the city-wide Black population, which was operationalized as ( $<41 \%$ or $\geq 41 \%$ ) since $41 \%$ of Philadelphia's residents are Black or African American alone [32]. Choropleth geographic maps of the social cohesion indicators and COVID-19 diagnosis rates were constructed using five natural breaks and overlaid by the ZIP codes with $\geq 41 \%$ Black or African American population. To test the association between social cohesion and COVID-19 diagnosis rate, a Poisson regression model with logarithm link function with the general population as the exposure variable was estimated. The base model included all the remaining social cohesion indicators simultaneously and adjusted for the density of COVID-19 testing sites and the spatial autocorrelation covariate. The latter two covariates are not presented in figures or results because they are not interpretable and for model robustness only. Incidence rate ratios (IRR) and $95 \%$ confidence intervals were produced. To determine whether the social cohesion variables are attenuated by covariates, additional adjustments were applied to the first model. Covariates included neighborhood socioeconomic position, crime index, proportion of renters moved, and proportion aged 18-24 and 25-34 and also Black racial composition because it is part of the multiplicative interaction model.

To test whether the association between social cohesion and COVID-19 diagnosis rates varied by distributions of Black neighborhood racial composition $(<41 \%$ or $\geq 41 \%$ ), a multiplicative interaction term between the two variables was included. The significance of the interaction was assessed using the Wald test statistic. Marginal predicted counts adjusted for all covariates were computed and graphed for interactions significant at $\alpha=p<0.05$ using the margins command. Stratified analysis was then conducted after identifying significant interaction results. Descriptive and multivariable analyses were conducted using STATA software version 15.1 .

\section{Results}

Univariate and Bivariate

The median (and interquartile range) of COVID-19 diagnosis rate in Philadelphia, PA, as of April 24, 2020 , was 101.4 per 10,000 general population (IQR=80.8-136.2) (results not displayed). There were 22 ZIP codes (48\%) where $\geq 41 \%$ of the population were Black or African American. In those ZIP codes, COVID-19 diagnosis rate per 10,000 general population was 119 (IQR=105.5-139.5), which was 35 cases higher than the overall rate of the remaining ZIP codes, which was 84 (IQR=70.5-107.6). 
The choropleth maps display supporting evidence that COVID-19 diagnosis rates are higher in ZIP codes where $\geq 41 \%$ of the population are Black or African American (Fig. 1). Moran's I test, however, revealed no statistically significant spatial clustering of COVID19 across Philadelphia ZIP codes $(I=-0.014, p=0.46$, results not displayed).

\section{Multivariable}

Civic and social participation was significantly associated with $11 \%$ lower COVID-19 diagnosis rates after adjustment for potential confounders (IRR $=0.89,95 \%$ $\mathrm{CI}=0.82,0.96$, Fig. 2). Higher neighborhood socioeconomic composition was associated with lower COVID19 rates $(\mathrm{IRR}=0.91,95 \% \mathrm{CI}=90,0.93)$. Higher black neighborhood racial composition was associated with $14 \%$ higher rates of COVID-19 diagnosis rates $(\mathrm{IRR}=1.14,95 \% \mathrm{CI}=1.09,1.20)$.

The multivariable multiplicative interaction models revealed that the direction and strength between civic and social participation (chi-square $=12.4$ $(\mathrm{df}=1), p=0.004)$ and collective engagement (chisquare $70.1(\mathrm{df}=1), p=0.000)$ on COVID-19 diagnosis rates were significantly different based on the Black neighborhood racial composition binary indicator (Fig. 3).

In stratified analyses, in ZIP codes where $\geq 41 \%$ of the population are Black or African American, higher feelings of belongingness was associated with $11 \%$ lower COVID-19 diagnosis rates (IRR $=0.89$, $95 \% \mathrm{CI}=0.79,1.00)$, but there was no significant

\section{City of Philadelphia}
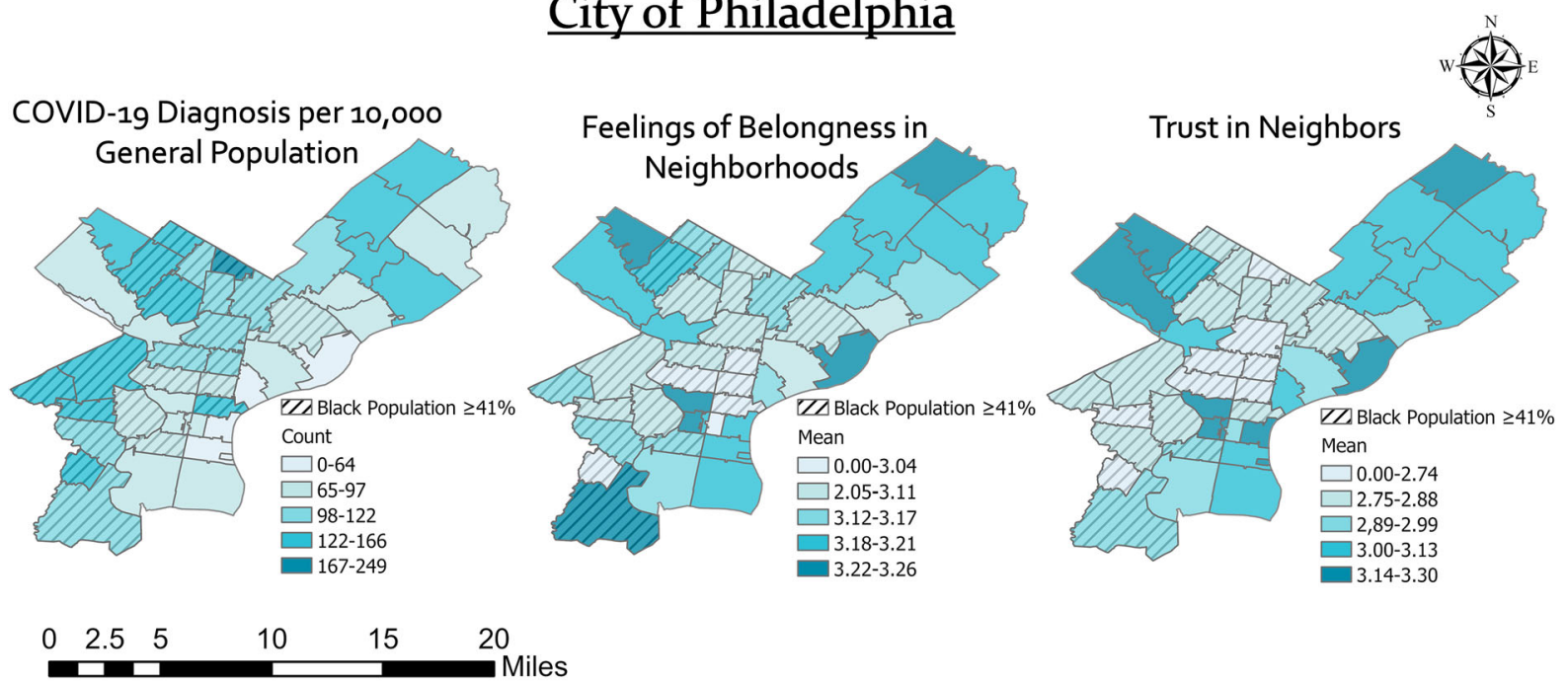

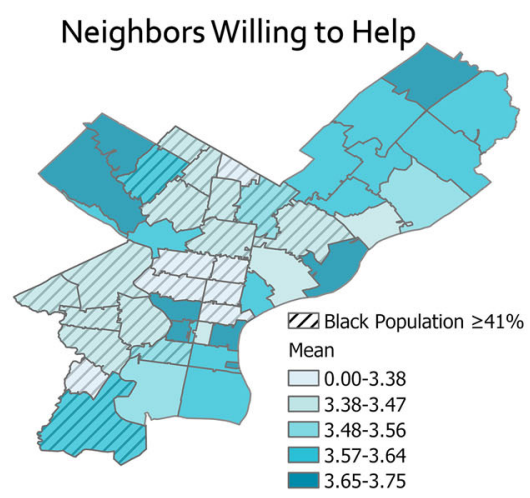

Fig. 1 Distribution of COVID-19 diagnosis rates and social cohesion indicators across Philadelphia, PA, ZIP codes $(N=46)$, April 2020. COVID-19 cases do not spatially cluster at the city-
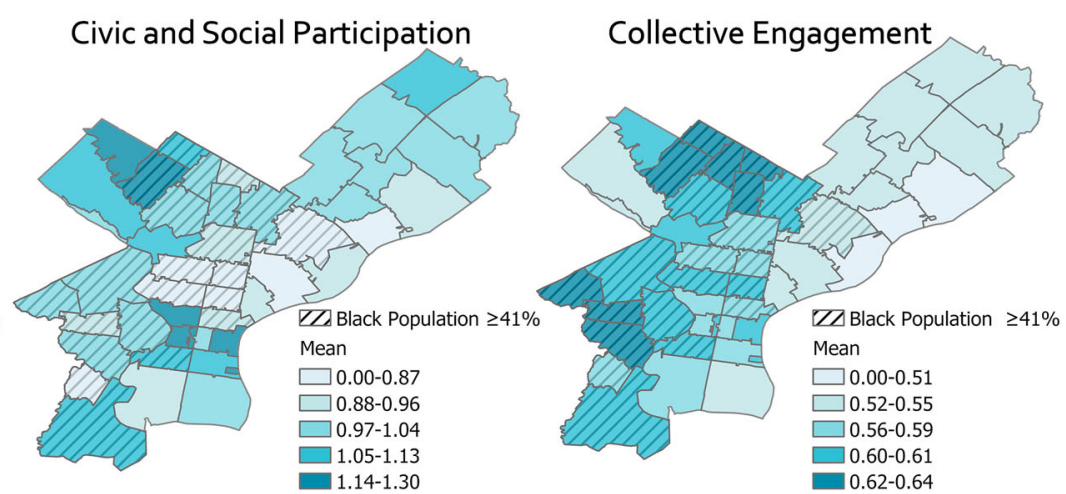

wide level but, rather, are higher in ZIP codes where the Black or African American population is $\geq 41$ percent. Social cohesion is also lower in those areas 
Fig. 2 Associations between social cohesion indicators and COVID-19 diagnosis rates across Philadelphia, PA, ZIP codes $(N=46)$, April 2020. ZIP codes with higher civic and social participation have $11 \%$ lower COVID-19 diagnosis rates in Philadelphia, PA. Higher Black racial neighborhood composition is associated with $14 \%$ higher COVID-19 diagnosis rates

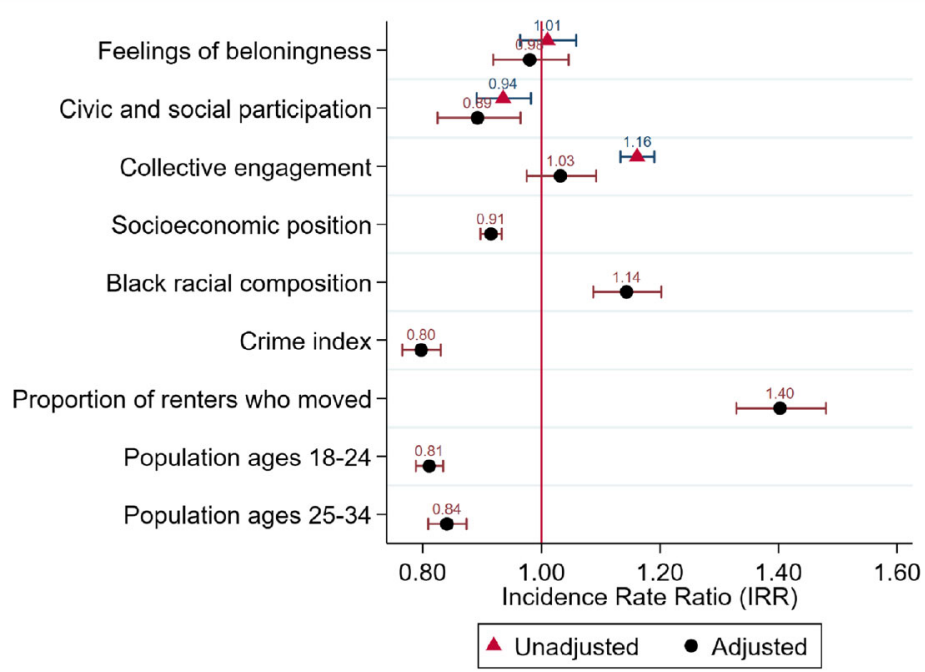

association in other neighborhoods (Fig. 4). In ZIP codes where $\geq 41 \%$ of the population are Black or

African American, higher collective engagement was associated with $18 \%$ higher COVID-19 diagnosis rates

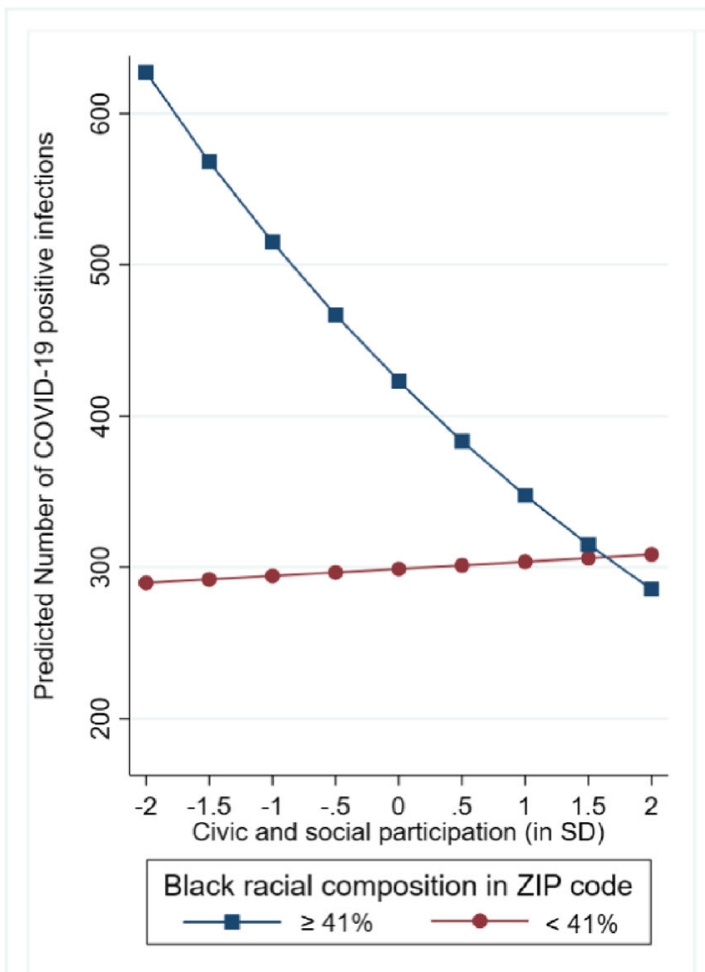

Fig. 3 Associations between two neighborhood social cohesion indicators and COVID-19 diagnosis rates are moderated by black neighborhood racial composition, Philadelphia, PA, ZIP codes $(N=46)$, April 2020. Higher civic and social participation and

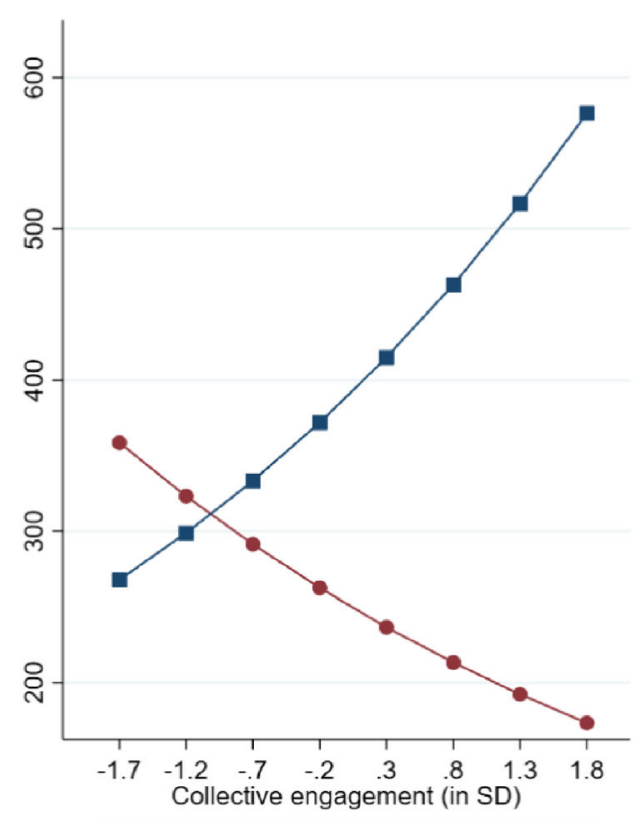

Black racial composition in ZIP code

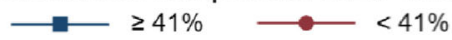

collective engagement have opposite associations with COVID19 diagnosis rates depending on whether ZIP codes where higher or lower than $41 \%$ of the population are Black or African American 
Fig. 4 Stratified analysis showing the association between neighborhood social cohesion and COVID-19 diagnosis rates, stratified by black neighborhood racial composition across Philadelphia, PA, ZIP codes $(N=46)$, April 2020. Higher collective engagement is associated with higher COVID-19 diagnosis rate in ZIP codes where $\geq 41$ percent of the population are Black or African American but lower COVIDdiagnosis rate in ZIP codes with more diverse racial composition

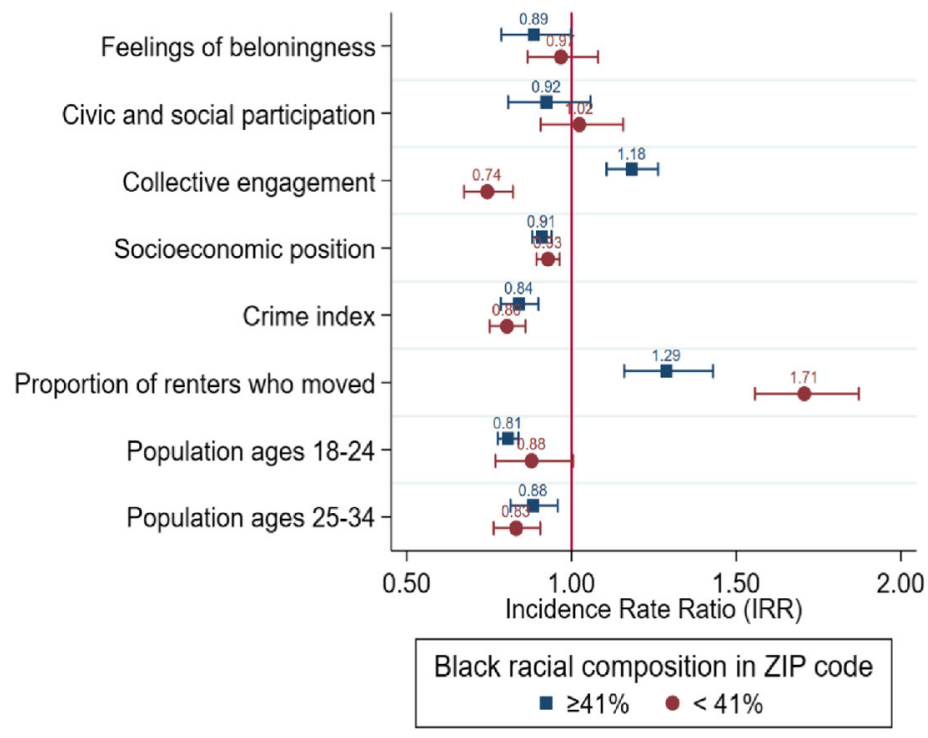

(IRR=1.18, 95\% CI=1.11, 1.26). In contrast, in ZIP codes $<41 \%$ Black or African American, higher collective engagement was associated with $26 \%$ lower COVID-19 diagnosis rates $(\mathrm{IRR}=0.74,95 \% \mathrm{CI}=0.67$, $0.82)$.

Surprisingly, in stratified analysis, civic and social participation was statistically unrelated to COVID-19 diagnosis rates in the context of other social cohesion indicators and covariates. Higher neighborhood socioeconomic composition was associated with lower COVID-19 diagnosis rates in both types of neighborhoods.

\section{Discussion}

This is the first ecological study at a local small-scale geographic level to document that civic and social participation and collective engagement are associated both positively and negatively with COVID-19 diagnosis. Recent studies have mostly assessed direct associations between COVID-19 and social cohesion or capital indicators. We advance the field with an interaction analysis where we found that some associations are not direct but that the size and directions vary by Black neighborhood racial composition. In ZIP codes with above average Black racial composition, lower COVID-19 diagnosis rates were associated with higher feelings of belongingness. In those same areas, higher COVID-19 diagnosis rates were associated with higher collective engagement - a measure of people working together to improve neighborhood conditions. In contrast, in areas below the city-wide average Black racial composition, lower COVID-19 diagnosis rates were associated with higher collective engagement, adjusting for other ZIP code socioeconomic and demographic covariates.

Several of our study findings are validated based on recently published work. In our study setting, higher area-level socioeconomic position was correlated with lower COVID-19 diagnosis, which is consistent with findings from another ZIP code-level study of New York City and Chicago [33]. Our findings revealed several nuances with respect to social cohesion, which is also consistent with published evidence to date [15]. First, some neighborhood social cohesion indicators were associated with higher rates of COVID-19 diagnosis, while others were associated with lower rates. Only civic and social participation, however, remained significantly related to lower COVID-19 diagnosis rates after adjustment for variables such as neighborhood socioeconomic position and crime. Second, COVID-19 diagnosis rates are higher in geographic areas where the Black racial composition is higher than the city-wide average, which in our study was in ZIP codes greater than or equal to forty one percent. This finding is also consistent with other ZIP code level studies from New York City and Chicago [33, 34] and with one other study which used county-level data and where racial composition was categorized based on values above or 
below thirteen percent - the US percent of the Black population [22].

What might our findings of direct associations and cross-over interaction patterns by neighborhood racial composition indicate for equity-based approaches to solutions? Public health prevention interventions may benefit from considering a segmentation approach [35] to deliver COVID-19 relief support based on distributions of neighborhood social cohesion and Black racial composition. Collective engagement traditionally involves in-person activities [26]. Within predominantly Black communities, inperson connectivity through faith-based institutions, social gatherings, and informal local activities such as block parties are conduits for coping, particularly during periods of stress and trauma, which was one effect of this pandemic.

In predominantly Black geographic communities, people may rely on higher in-person connectivity as a strategy for communication and dissemination of information or resources needed to survive. The fast spread of COVID and inconsistent public health information earlier in the pandemic happened without sufficient time for people to prepare or adjust their behaviors and accumulate resources. It is also plausible in those areas that people lacked or were not fully utilizing virtual communication modes within their social networks. In one national survey, for instance, only $64 \%$ of Black people compared to $78 \%$ of White people used email or messaging services to communicate with others during the COVID-19 pandemic [36]. Communication differences are not just a matter of preferences but socioeconomic status resources, which are lower in predominantly Black geographic communities [23]. Some cities offer low-cost or free internet options in low-income areas [37] which often overlap with predominantly Black geographic communities. However, fear of surveillance, historical institutional racism, and mistrust of big business and government will need to be addressed [38] in interventions designed to increase uptake of technology and reduce disparities.

We recommend a few strategies for social cohesionbased interventions based on a modified segmentation approach [35] that prioritizes predominantly Black geographic communities. If these findings hold in other studies, government policies can be implemented to encourage greater public health-corporate-community partnerships that use community volunteers to deliver food to those in quarantine or who cannot leave their homes $[39,40]$. This sort of partnership might be one pathway to improve collective engagement in these areas.

Interventions should be intentional about the types of social participation to increase. At the city-level, one strategy could be to expand financial and political investment in public health-faith collaborations with Historically Black Churches in predominantly Black areas [41]. A biomedical intervention should combine wraparound social services and expanded access to food distribution in the community. Many churches in predominantly Black neighborhoods are trusted because they have been providing social and health services to residents before the pandemic emerged [42]. Churches also provide spiritual and religious support to residents. Therefore, spiritual and religious support on aggregate within a geographic community may help create and sustain norms of caring and neighbors helping neighbors [43] needed to flatten the COVID-19 infection curve and help all people thrive beyond this pandemic.

At the federal level, greater funding and investment in religious or faith-based organizations within Black neighborhoods are pivotal. One specific approach could be to equip these organizations to disburse the CARES Act funding. These institutions play critical roles in political processes and that influence the uptake of public health initiatives, especially among hard-to-reach vulnerable populations like the elderly and people with housing insecurity [44]. Federal commitment of funding is a crucial ingredient to build trust and community buyin not only to promote compliance of public health ordinances now but to sow the seed that may build trust to achieve high uptake of the COVID-19 vaccinations when implemented in the general population.

Public behavior including wearing masks is a function of social psychological processes such as identity, neighborhood attachment, and supportive social group norms. Public health prevention messaging strategies might make use of people's sense of belongingness to their communities since we found a small but salutary association with COVID-19 diagnosis rates. Messages should also communicate shared concern for protecting loved ones and solidarity among people $[45,46]$ within their geographic community.

\section{Limitations}

No study is without limitations. COVID-19 diagnoses surveillance data in this city, as is the case for data in several other areas across the USA, were not available 
by race. Therefore, we cannot make inferences about racial inequalities in COVID-19 diagnosis rates. Accurate measures of physical distancing and other behavioral exposure factors (e.g., wearing masks) or measures of participation in ordinances (e.g., quarantine) do not currently exist for this city. These are likely mediators that would explain some proportion of any associations that we found. Although we controlled for the density of COVID-19 testing sites, testing policies in April 2020 were driven by infection need distribution, and therefore, COVID-19 cases were likely under-counted in more disadvantage areas. The modifiable areal unit problem may exist such that if these analyses were replicated at other geographic levels (e.g., census tract), results may not hold. In this study, we used the ZIP code, which in Philadelphia and other cities is most granular level of data publicly available.

The social cohesion indicators, while collected in 20182019, are cross-sectional. Although there is some temporality between the exposures and outcome variable, we still cannot draw causal inferences with COVID-19 diagnosis rates in April 2020. Indicators of social cohesion should be collected in the context of COVID when people are social distancing and engaging with others online. For instance, civic and social participation reflects pre-pandemic behavior and does not account for the type of engagement people were limited to when physical distancing and quarantine ordinances were in effect. The collective engagement question in this household survey is generic and part of a scale created more than two decades ago in the context of studies to explain crime rates [47]. Rapid telephone surveys could be conducted during pandemics to ask people how they have engaged with neighbors, potentially through questions like during the pandemic "have you helped your neighbor with grocery shopping?" or "have you checked in with your neighbor to see if they needed help?" Other unobserved confounders like population mobility rates within neighborhoods might bias the estimates downward. However, those data are not currently available. Timing between exposure and interests are also important [15]. While we captured COVID-19 infections at one time point, it is possible that social cohesion might have a stronger relationship with COVID-19 diagnosis [21] earlier in the pandemic when people were still figuring out the effects and needed help. Despite these limitations, we used population-based surveillance COVID-19 data and multiple reliable indicators of social cohesion across a large geographically diverse local setting while controlling for several confounders.

\section{Conclusion}

The epidemiology of COVID-19 reveals inequalities in diagnosis and mortality rates that are higher in geographic areas with higher Black racial composition. Neighborhood social cohesion is associated with COVID-19 diagnosis rates in complex ways, and some associations vary by Black neighborhood racial composition. Future ecological studies at the local levels in other US cities are needed to examine the association between social cohesion and racespecific COVID-19 diagnosis rates. We also need multilevel studies that link area-level and individual risk and protective factors among those diagnosed with and among those who recovered from COVID-19. Longitudinal studies that can assess the relationship between social cohesion and COVID-19 diagnosis rates at different time points will be crucial to understand how communities are responding to trends in infection. We need also studies that can test causal linkages between social cohesion and intentions to get vaccinated or vaccine hesitancy through mediating pathways that include COVID-19 diagnosis and other prevention protocols.

Thus far, several ecological studies hypothesize pathways, or mechanisms such as social cohesion would influence COVID-19 diagnosis, which includes respect and obedience for social distancing and other public health prevention ordinances, higher empathy, diffusions of information, and neighbors taking responsibility for others. However, these pathways were often not tested empirically. Therefore, large probability sample studies that can assess plausible mechanisms are critical to identify behavioral, economic, social, and biological pathways through which social cohesion influences COVID-19 transmission and, in the future, vaccination likelihood.

Acknowledgements Yusuf Ransome was supported by the National Institute of Mental Health under award number [K01MH111374]. Bisola O. Ojikutu was supported by Harvard University Center for AIDS Research grant under award number [P30 AI060354] and the National Institute of Mental Health under award number [K23MH107316]. This content is solely the responsibility of the authors and does not necessarily represent the official views of the National Institutes of Health. The funders had no role in the design and conduct of the study; collection, management, analysis, and interpretation of the data; preparation, review, or approval of the manuscript; and decision to submit the manuscript for publication.

\section{Declarations}

Ethics Approval This article does not contain any studies with human participants performed by any of the authors. 


\section{References}

1. The COVID Tracking Project. The COVID racial data tracker 2020. Available from: https:/covidtracking. com/race. Accessed 4 July 2020.

2. Oppel Jr RA, Gebeloff R, Lai RK, Wright W, Smith M. The fullest look yet at the racial inequity of coronavirus New York, NY: The New York Times; 2020 [updated July 5 2020]. Available from: https://www.nytimes. com/interactive/2020/07/05/us/coronavirus-latinos-africanamericans-cdc-data.html2020/07/05/us/coronavirus-latinosafrican-americans-cdc-data.html. Accessed 3 Aug 2020.

3. Millett GA. New pathogen, same disparities: why COVID19 and HIV remain prevalent in U.S. communities of colour and implications for ending the HIV epidemic. J Int AIDS Soc. 2020;23(11):e25639. https://doi.org/10.1002 /jia2.25639.

4. Bambra C, Riordan R, Ford J, Matthews F. The COVID-19 pandemic and health inequalities. J Epidemiol Community Health. 2020;0:jech-2020-214401. https://doi.org/10.1136 /jech-2020-2144012020-214401.

5. Egede LE, Walker RJ. Structural racism, social risk factors, and COVID-19-a dangerous convergence for Black Americans. N Engl J Med. 2020. https://doi.org/10.1056 /NEJMp2023616.

6. Hooper MW, Nápoles AM, Pérez-Stable EJ. COVID-19 and racial/ethnic disparities. J Am Med Assoc. 2020;24:2466-7. https://doi.org/10.1001/jama.2020.8598.

7. Yancy CW. COVID-19 and African Americans. J Am Med Assoc. 2020;323(19):1891-2. https://doi.org/10.1001 /jama.2020.6548.

8. Chowkwanyun M, Reed AL. Racial health disparities and COVID-19 - caution and context. N Engl J Med. 2020. https://doi.org/10.1056/NEJMp2012910.

9. Cattell V. Poor people, poor places, and poor health: the mediating role of social networks and social capital. Soc Sci Med. 2001;52(10):1501-16. https://doi.org/10.1016/S02779536(00)00259-8.

10. Macinko J, Starfield B. The utility of social capital in research on health determinants. Milbank Q. 2001;79(3):387427.

11. Kawachi I, Berkman L. Social cohesion, social capital and health. In: Kawachi I, Berkman L, Glymour M, editors. Social Epidemiology. 2nd ed. New York, NY: Oxford University Press; 2014. p. 291-319.

12. Fonseca X, Lukosch S, Brazier F. Social cohesion revisited: a new definition and how to characterize it. Innovation: The European Journal of Social Science Research. 2018;32(2): 231-53. Epub 16 Jul 2018. https://doi.org/10.1080 /13511610.2018.1497480.

13. Chuang Y-C, Huang Y-L, Tseng K-C, Yen C-H, Yang L-h. Social capital and health-protective behavior intentions in an influenza pandemic. PLOS ONE. 2015;10(4):e0122970. https://doi.org/10.1371/journal.pone.0122970.

14. Jung M, Lin L, Viswanath K. Associations between health communication behaviors, neighborhood social capital, vaccine knowledge, and parents' H1N1 vaccination of their children. Vaccine. 2013;31(42):4860-6. https://doi. org/10.1016/j.vaccine.2013.07.068.
15. Wu C. Social capital and COVID-19: a multidimensional and multilevel approach. Chinese Sociological Review. 2020;53: 1-28. https://doi.org/10.1080/21620555.2020.1814139.

16. Ding W, Levine R, Lin C, Xie W. Social distancing and social capital: why U.S. counties respond differently to COVID-19: SSRN Working Paper; 2020 [updated 12 June 2020]. Available from: https://papers.ssrn.com/sol3 /papers.cfm?abstract_id=3624495. Accessed 5 Jan 2021.

17. Varshney LR, Socher R. COVID-19 growth rate decreases with social capital. medRxiv. 2020. https://doi.org/10.1101 /2020.04.23.20077321.

18. Borgonovi F, Andrieu E. Bowling together by bowling alone: social capital and COVID-19: Center for Economic Policy Research (CEPR) Press; 2020. Available from: https://dagliano.unimi.it/wp-content/uploads/2020/05 /CovidEconomics 17.pdf\#page=78. Accessed 5 Jan 2020.

19. Makridis CA, Cary W. Ties that bind (and social distance): how social capital helps communities weather the COVID19 pandemic: SSRN Working Paper; 2020 [updated 13 August 2020]. Available from: https://papers.ssrn.com/sol3 /papers.cfm?abstract_id=3592180. Accessed 5 Jan 2021.

20. Campo-Arias A, Perdomo-Rojas JA, Caballero-Domínguez CC. Social capital and perceived stress related to coronavirus disease in Colombia. J Epidemiol Community Health. 2020;0:jech-2020-215005. https://doi.org/10.1136/jech2020-215005.

21. Fraser T, Aldrich DP, Page-Tan C. Bowling alone or masking together? the role of social capital in excess death rates from COVID19: SSRN; 2020 [updated December 11 2020]. Available from: https://papers.ssrn.com/sol3/papers. cfm?abstract id=3744251. Accessed 5 Jan 2020.

22. Millett GA, Jones AT, Benkeser D, Baral S, Mercer L, Beyrer C, et al. Assessing differential impacts of COVID-19 on Black communities. Ann Epidemiol. 2020. https://doi.org/10.1016/j. annepidem.2020.05.003.

23. Williams DR, Collins C. Racial residential segregation: a fundamental cause of racial disparities in health. Public Health Rep. 2001;116(5):404-16.

24. Wilson WJ. The truly disadvantaged: the inner city, the underclass, and public policy. Chicago, IL: University of Chicago Press; 2012.

25. Kawachi I, Kennedy BP, Lochner K, Prothrow-Stith D. Social capital, income inequality, and mortality. Am J Public Health. 1997;87(9):1491-8.

26. Dean LT, Hillier A, Chau-Glendinning H, Subramanian SV, Williams DR, Kawachi I. Can you party your way to better health? a propensity score analysis of block parties and health. Soc Sci Med. 2015;138:201-9. https://doi. org/10.1016/j.socscimed.2015.06.019.

27. Azavea. OpenDataPhilly Philadelphia, PA: OpenDataPhilly. org; 2015. Available from: OpenDataPhilly.org. Accessed 8 Aug 2015.

28. Public Health Management Corporation Community. Southeastern Pennsylvania Household Health Survey Philadelphia, PA; 1983. Available from: http://www. chdbdata.org/index.php. Accessed 15 Nov 2015.

29. Ransome Y, Galea S, Pabayo R, Kawachi I, Braunstein S, Nash D. Social capital is associated with late HIV diagnosis: an ecological analysis. J Acquir Immune Defic Synd. 2016;73(2):213-21. 
30. Ransome Y, Kawachi I, Dean LT. Neighborhood social capital in relation to late HIV diagnosis, linkage to HIV care, and HIV care engagement. AIDS Behav. 2016;21(3):891904.

31. Goswami ND, Schmitz MM, Sanchez T, Dasgupta S, Sullivan P, Cooper H, et al. Understanding local spatial variation along the care continuum: the potential impact of transportation vulnerability on HIV linkage to care and viral suppression in high-poverty areas, Atlanta, Georgia. $J$ Acquir Immune Defic Synd. 2016;72(1):65-72.

32. The PEW Charitable Trusts. Philadelphia 2019: the state of the city Philadelphia, PA: PEW Foundation; 2019. Available from: https://www.pewtrusts.org/-/media/assets/2019/10 /sotc_2019.pdf2019/10/sotc_2019.pdf. Accessed 4 July 2020.

33. Maroko AR, Nash D, Pavilonis BT. COVID-19 and inequity: a comparative spatial analysis of New York City and Chicago hot spots. J Urban Health. 2020;97(4):461-70. https://doi.org/10.1007/s11524-020-00468-0.

34. DiMaggio C, Klein M, Berry C, Frangos S. Black/African American communities are at highest risk of COVID-19: spatial modeling of New York City ZIP Code-level testing results. Ann Epidemiol. 2020;51:7-13. https://doi. org/10.1016/j.annepidem.2020.08.012.

35. Villalonga-Olives E, Wind T, Kawachi I. Social capital interventions in public health: a systematic review. Soc Sci Med. 2018;212:203-18.

36. Anderson M, Vogels EA. Americans turn to technology during COVID-19 outbreak, say an outage would be a problem Washington, DC: Pew Research Center; 2020. Available from: https://www.pewresearch.org/facttank/2020/03/31/americans-turn-to-technology-duringcovid-19-outbreak-say-an-outage-would-be-aproblem/2020/03/31/americans-turn-to-technology-duringcovid-19-outbreak-say-an-outage-would-be-a-problem/. Accessed 31 Mar 2020.

37. Philadelphia Co. PHLConnectED: connecting K-12 student households to digital access with internet, laptops, and training. Philadelphia, PA; 2020. Available from: https://www. phila.gov/programs/phlconnected/. Accessed 5 Jan 2021.

38. Sabatello M, Jackson Scroggins M, Goto G, Santiago A, McCormick A, Morris KJ, Daulton CR, Easter CL, Darien G. Structural racism in the COVID-19 pandemic: moving forward. Am J Bioeth. 2020;21(5):1-31.

39. Sampson K. Walmart and Nextdoor launch new "neighbors helping neighbors" program. San Francisco, CA: Nextdoor Inc; 2020. Available from: https://about.nextdoor. com/walmart-and-nextdoor-launch-new-neighbors-helpingneighbors-program/. Accessed 4 July 2020.
40. Kobokovich AL, Hosangadi D, Rivers C. Supporting social distancing for COVID-19 mitigation through communitybased volunteer networks. Am J Public Health. 2020;0(0): e1-e2. https://doi.org/10.2105/ajph.2020.30574 02020.305740 .

41. Governor Andrew M. Cuomo Press Office. Amid ongoing COVID-19 pandemic, Governor Cuomo launches new initiative to expand access to testing in low-income communities of color. Albany, NY; 2020. Available from: https:/www.governor.ny.gov/news/amid-ongoing-covid19-pandemic-governor-cuomo-launches-new-initiativeexpand-access-testing-low. Accessed 3 Aug 2020.

42. Brewer LC, Williams DR. We've come this far by faith: the role of the Black church in public health. Am J Public Health. 2019;109(3):385-6. https://doi.org/10.2105 /ajph.2018.304939.

43. Rodriguez A. Coronovirus contact tracers remain critical in stemming the spread, lending a helping hand. Boston, MA: Boston CBS; 2020. Available from: https://boston.cbslocal. com/2020/08/17/coronavirus-contact-tracers-criticalstopping-spread/2020/08/17/coronavirus-contact-tracerscritical-stopping-spread/. Accessed 17 Aug 2020

44. Kiser M, Lovelace K. A national ntwork of public health and faith-based organizations to increase influenza prevention among hard-to-reach populations. Am J Public Health. 2019;109(3):371-7. https://doi.org/10.2105 /AJPH.2018.304826.

45. Drury J, Carter H, Cocking C, Ntontis E, Tekin Guven S, Amlôt R. Facilitating collective psychosocial resilience in the public in emergencies: twelve recommendations based on the social identity approach. Front Public Health. 2019;7(141). https://doi.org/10.3389/fpubh.2019.00141.

46. Bonell C, Michie S, Reicher S, West R, Bear L, Yardley L, et al. Harnessing behavioural science in public health campaigns to maintain 'social distancing' in response to the COVID-19 pandemic: key principles. J Epidemiol Community Health. 2020;74(8):617-9. https://doi. org/10.1136/jech-2020-214290.

47. Sampson R, Raudenbush S, Earls F. Neighborhoods and violent crime: a multilevel study of collective efficacy. Science. 1997;277:918-24. https://doi.org/10.1126 /science.277.5328.918.

Publisher's Note Springer Nature remains neutral with regard to jurisdictional claims in published maps and institutional affiliations. 